\title{
DaFT-Zertifikat im Rahmen eines MOOC Deutsch für den Tourismus
}

\section{DaFT certificate as part of a MOOC German for Tourism}

\author{
Gloria Isabel Bosch Roig ${ }^{1}$ (1) https://orcid.org/0000-0003-0291-4204 \\ Universidad de las Islas Baleares \\ Edifici Arxiduc Lluís Salvador · Ctra. de Valldemossa, Km 7.5 • 07122 · Palma de Mallorca · Baleares (Spain)
}

\section{ZUSAMMENFASSUNG}

DaFT (Deutsch als Fremdsprache für Tourismus) wird in formellen bzw. akademischen berufsbildenden Kontexten vermittelt und dient somit primär der Bewältigung und Koordination beruflicher Abläufe und Handlungen seitens der Arbeitnehmer, je nach ihrer betrieblichen Funktion im Tourismusbetrieb. Das Ziel des DaFT-Unterrichts ist letztlich die Förderung einer beruflichen sprachübergreifenden Handlungsfähigkeit und einer berufsbezogenen kommunikativen Kompetenz als Registerkompetenz in internationalen bzw. interkulturellen Zusammenhängen bzw. Kontexten. DaFT ist insofern eine Interaktionssprache, die Tourismusstudenten auf reale Interaktionssituationen im Berufsleben mit kulturunterschiedlichen Sprechern (des Deutschen) vorbereiten soll. Aus diesem Grund gehen wir davon aus, dass rezeptive Fähigkeiten wie das Hörverstehen und insbesondere die mündliche Sprachproduktion im Hauptfokus des DaFT-Unterrichts stehen sollen.

Der Online-Grundkurs (MOOC) Deutsch für Tourismus der Universität der Balearen ist in diesem Lehr- und Lernkontext entstanden und hat sich zum Ziel gemacht, den Tourismus-Studierenden sowie den autonomen Deutschlernern, die im Tourismusbereich tätig sind, bei der Entwicklung und Überprüfung dieser Fähigkeiten zu helfen. Nun steht das Projekt zur Entwicklung eines auf diese im MOOC geforderten Fähigkeiten und Sprachkompetenzen basierenden DaFT-Tests im Mittelpunkt unseres Beitrags.

Schlüsselwörter: Projekt, DaFT-Test, Test-Entwicklung, DaFT MOOC

\section{ABSTRACT}

German as a foreign language for tourism (DaFT, from the German) is taught in academic and vocational training contexts and is thus primarily aimed at those who work in different areas of the tourism industry, coordinating professional processes and activities. DaFT classes aim to foster linguistic ability in this professional field and develop communicative skills, including those of register, in international, intercultural contexts. In this sense, DaFT is used in interactions, and the classes have to prepare students of Tourism to be able to interact in real situations with German speakers from different cultures. For this reason, we assume that DaFT lessons should focus on the skills of aural comprehension and speaking,

The basic online course (MOOC) German for Tourism at the University of the Balearic Islands originated in this teaching- learning context with a view to helping students of Tourism and other learners of German who work in the tourism industry to put their skills to the test. This article presents a project for the drawing up of a DaFT test based on the language abilities and competences covered in the MOOC.

Keywords: Project, DaFT test, test development, DaFT MOOC

\section{Einleitung}

Ausgehend von den Merkmalen der Berufssprachen kann Deutsch als Fremdsprache für den Tourismus (DaFT) nicht als eine Fachsprache, sondern als eine weitere Berufssprache verstanden werden (Bosch-Roig, 2018). Fachsprachen dienen primär der Wissensvermittlung und sind an Experten gebunden. Sie benötigen keinen 
Semantisierungskontext und sind durch die Eindeutigkeit und Klarheit der Begriffe und Aussagen gekennzeichnet, die dem Ziel der Kommunikation in einem begrenzbaren Kommunikationsbereich zwischen Fachexperten dienen. Berufssprachen entstehen jedoch in einem unterrichtlichen Kontext und ergeben sich aus der Notwendigkeit einer beruflichen Kommunikationssituation, um die Anforderungen des realen beruflichen Lebens erfolgreich zu bewältigen (ebd.)

Wir gehen davon aus, dass eine Berufssprache kein separates Register ist, sondern in höherem oder geringerem Grad Merkmale der drei Register (Alltags-, Fach-, und Bildungssprache) aufweist. Bildungssprachen sind für die Bewältigung abstrakt-/kognitiver Aufgaben sowie für den Wissenserwerb und die Wissensvermittlung besonders wichtig. Sprachhandlungen wie das Benennen, Beschreiben, Erklären, Argumentieren, Informieren, Empfehlen u.a. sind abstrakte, komplexe sprachlich-kognitive Prozesse, die dem Register der Bildungssprache zugeschrieben werden können. Dementsprechend verlangen Bildungssprachen einen expliziten, präzisen, strukturierten und objektiven Gebrauch (Efing, 2014). Die Spezifität der Berufssprache hängt jedoch von ihrer Position auf dem Kontinuum zwischen Alltags-, Bildungs- und Fachsprache ab. Je mehr sie sich dem äußersten Standpunkt der Fachsprache nähert, desto spezifischer und kontextunabhängig ist sie (ebd.). Eine Berufssprache ist aus Prinzip handlungsorientiert, sie wird in formellen berufsbildenden Kontexten vermittelt und erlernt, und dient der Bewältigung und Koordination beruflicher Abläufe und Handlungen, die komplexe sprachlichkognitive Prozesse voraussetzen. Das Ziel des DaFT-Unterrichts ist demnach die Förderung einer beruflichen Handlungsfähigkeit und einer berufsbezogenen kommunikativen Kompetenz in internationalen Zusammenhängen. DaFT ist eine berufshandlungsorientierte Interaktionssprache.

In einer völlig vernetzten Welt, die durch die zunehmende Mobilität ihrer Bewohner gekennzeichnet ist, sei es für Freizeit und Vergnügen, für die Arbeit oder zum Überleben, ist es logisch zu denken, dass Sprachen „unfreiwillig“ gelernt werden müssen (Long, 2014: 3). Betrachten wir zum Beispiel den Fall des Deutschen in Spanien, eine der Fremdsprachen, die in den letzten Jahren vor allem aufgrund wirtschaftlicher und beruflicher Faktoren eine große Nachfrage (Bosch-Roig, 2016, 2018) erlebt hat. Zweifellos kann Deutsch heute als eine wichtige Arbeitssprache bzw. Berufssprache angesehen werden. Sein Beschäftigungsnutzen wird nicht nur von Fachleuten anerkannt, die an Reisen und Arbeit im Land interessiert sind, sondern auch von einer Vielzahl von Arbeitnehmern, die vom spanischen Tourismussektor und vom mächtigen deutschen Emissionsmarkt, insbesondere nach den Balearen und den Kanarischen Inseln, abhängig sind.

Die gegenwärtige Realität zwingt uns, die Prämisse zu akzeptieren, dass die Beschäftigungsfähigkeit am Arbeitsplatz zunehmend mit einer zunehmenden Ausbildung und Spezialisierung der Arbeitssuchenden verbunden ist und das betrifft die Fremdsprachenkenntnisse insbesondere. Da die Ressourcen als auch die Lernzeit begrenzt sind, erhöht sich die Notwendigkeit einer Optimierung dieser Komponenten. Das Angebot von Fremdsprachen innerhalb aller akademischen Studien, auch außerhalb der akademischen Welt, sollte aus diesem Grund garantiert sein und auf einen tatsächlichen Bedarf, d.h. auf eine Analyse der unmittelbaren Umgebung reagieren. Dieses „unmittelbare Umfeld“ bezieht sich auf den engsten und direktesten Kontext der Studierenden, in erster Linie auf die lokale und regionale Nachfrage, auf die Unternehmen sowie auf die öffentlichen und privaten Institutionen, die an den wirtschaftlichen und sozialen Prozessen beteiligt sind.

Als Beispiel möchte ich auf die Balearen verweisen, insbesondere auf die Insel Mallorca, auf der der touristische Wirtschaftssektor dominiert. Deutschland ist mit mehr als 4 Millionen Touristen pro Jahr der erste Entsendemarkt auf die Balearen (IBESTAT, 2019). In gleicher Weise gibt es auch immer mehr deutsche Unternehmen, nicht nur Tourismusunternehmen, die auf den Inseln ansässig sind. Die deutsche Sprache ist 
derzeit aus diesem Grund eine sehr wichtige Berufssprache in diesem geografischen Gebiet. Wir müssen jedoch das begrenzte Ausbildungsangebot dieser Sprache im akademischen Bereich der Universität feststellen, was zu einer paradoxen Situation führt: eine akademische Überqualifizierung zusammen mit einer geringen sprachlichen Ausbildung. Das derzeitige Sprachtraining der Studierenden des Studiengangs Tourismus garantiert nicht ihre Wettbewerbsfähigkeit auf dem Arbeitsmarkt der Insel. Dieser Umstand ist nicht nur auf den Balearen anzutreffen: die Ausbildung in Fremdsprachen in Spanien war schon immer und ist bis heute problematisch (Eurostat, 2019).

Das Bachelor-Studium in Tourismus und das Bachelor-Doppelstudium Tourismus-BWL, die von der Fakultät für Tourismus der Universität der Balearen angeboten werden, umfassen nur drei Pflichtkurse in Deutsch innerhalb drei Semester, die lediglich zu einem niedrigen B1-Niveau führen und es den Studenten unmöglich macht, komplexe Aufgaben im beruflichen Umfeld zu lösen. Tourismusstudenten wird daher von der Fakultät empfohlen, vor der Einschreibung in den ersten Deutschkurs selbst ein vollständiges A1-Niveau zu absolvieren. Diese wichtige Voraussetzung wird jedoch nicht immer erfüllt, so dass wir in den ersten Kursen Studenten ohne Vorkenntnisse antreffen. Um dieses Problem zu lösen, haben wir beschlossen, ein MOOC (Massive Open Online Course) für DaFT-Anfänger zu entwickeln, d.h. einen Kurs für die Stufen A1-A2, und auf diese Weise unseren Studenten den Zugang zu den Vorkenntnissen zu erleichtern, die erforderlich sind, um die Pflichtfächer zu absolvieren.

Ein auf der digitalen Plattform der Universität gehostetes MOOC mit direktem Zugriff über das virtuelle Klassenzimmer garantiert den Studenten Zugang und bietet ihnen genügend Flexibilität und Vernetzung, um ihren eigenen Lernprozess zu steuern und zu organisieren.

Nach einer kurzen Kontextualisierung und Vorstellung des bereits laufenden MOOC an der Universität der Balearen und auf der Plattform Miríadax haben wir uns nun in diesem Zusammenhang und auf unserem OnlineKurs basierend vorgenommen, das künftige Projekt zur Entwicklung eines DaFT-Tests vorzustellen. Wir möchten jedoch an diesem Punkt vorwegnehmen, dass unser Ziel nicht darin besteht, Hypothesen aufzustellen und zu überprüfen, sondern lediglich Überlegungen zu erläutern, welche dem Entstehungsgrund dieses Projekts zugrunde liegen.

\section{Aufgaben- und Inhaltsorientierung im DaFT-Unterricht als methodologischer Ansatz der Hand- lungs- und berufsbezogenen Kompetenzorientierung}

Das Prinzip der Handlungs- und Kompetenzorientierung steht im Zentrum der aktuellen Fachdiskussion über didaktisch-methodische Fragen, wie dem Gemeinsamen europäischen Referenzrahmen zu entnehmen ist (Europarat, 2001). Relevante handlungsorientierte Aufgaben (des Berufslebens) sollen die Lernenden und Studierenden dazu befähigen, Probleme gemeinsam auszuhandeln und sprachlich zu lösen. Der aufgaben- und inhaltsorientierte Lernansatz eignet sich aus diesem Grund besonders gut (Bosch-Roig, 2013b, 2018), denn dabei werden formelle und kommunikative Aspekte der Sprache eng miteinander verknüpft, um das primäre kommunikative Ziel zu erreichen. Im handlungsorientierten DaFT-Unterricht, und das betrifft auch die Prüfungsaufgaben, werden die Lernenden und Studierenden mit Situationen und Themen des TourismusBerufslebens konfrontiert, die für sie und ihre professionelle Zukunft relevant und von Bedeutung sind.

Die Handlungsorientierung als zentrales Prinzip des DaFT-Unterrichts setzt aber voraus, dass die Lernenden und Studierenden auch über verschiedene Kompetenzen verfügen, die sie befähigen, die Aufgaben erfolgreich zu lösen. Kompetenzorientierung geht also einen Schritt weiter. Fremdsprachliche Kompetenzen werden im 
Gemeinsamen europäischen Referenzrahmen für Sprachen (GER) beschrieben und gelten mittlerweile ,als zentrales Instrument der Qualitätsentwicklung und Qualitätssicherung im Bereich des Testens, Prüfens und Evaluierens fremdsprachlicher Kompetenzen“ (Grotjan, Kleppin, 2015:21). Die Globalskala des GER (Europarat, 2001:35) beschreibt die Kompetenzniveaus A1 bis C1. Wenn heutzutage Sprachkurse und Sprachprüfungen angeboten werden, dann werden diese an die GER-Kompetenzniveaus angepasst (Grotjan, Kleppin, 2015), wobei der Kompetenzzuwachs nicht nur als ein „Vorankommen auf einer vertikalen Skala“ verstanden wird, denn Lernende und Studierende können auch Fortschritte machen, wenn sie ihre Sprachkompetenzen auf einer bestimmten Stufe verbreitern und vertiefen (Europarat, 2001:29). Im GER werden Hörverstehen, Leseverstehen, Sprechen, Hör-Sehverstehen und Sprachmittlung als kommunikative Aktivitäten bezeichnet und anhand der Prozessmerkmale (Produktion, Rezeption, Interaktion und Sprachmittlung), der Modalität (mündlich oder schriftlich) und der Art der Wahrnehmung (auditiv oder visuell) beschrieben. So können die traditionellen (Grund)Fertigkeiten und spezifischere Aktivitäten genauer beschrieben werden (Goethe Institut, 2017).

\begin{tabular}{|c|c|c|c|c|}
\hline \multicolumn{3}{|c|}{$\begin{array}{l}\text { Globalskala für die Niveaustufen A1 - A2 } \\
\text { (Europarat 2001:35) }\end{array}$} & \multicolumn{2}{|c|}{$\begin{array}{l}\text { Globalskala für die Niveaustufen A1-A2 } \\
\text { Deutsch als Fremdsprache für Tourismus }\end{array}$} \\
\hline $\begin{array}{l}\text { Elementare } \\
\text { Sprachverwendung }\end{array}$ & A1 & $\begin{array}{l}\text { Kann vertraute, alltägliche } \\
\text { Ausdrücke und ganz } \\
\text { einfache Sätze verstehen } \\
\text { und verwenden, die auf die } \\
\text { Befriedigung konkreter } \\
\text { Bedürfnisse zielen. } \\
\text { Kann sich und andere } \\
\text { vorstellen und anderen } \\
\text { Leuten Fragen zu ihrer } \\
\text { Person stellen z.B. wo sie } \\
\text { wohnen, etc. und kann auf } \\
\text { Fragen dieser Art Antwort } \\
\text { geben. } \\
\text { Kann sich auf einfache Art } \\
\text { verständigen, wenn die } \\
\text { Gesprächspartnerinnen oder } \\
\text { Gesprächspartner langsam } \\
\text { und deutlich sprechen und } \\
\text { bereit sind zu helfen. }\end{array}$ & A1 & 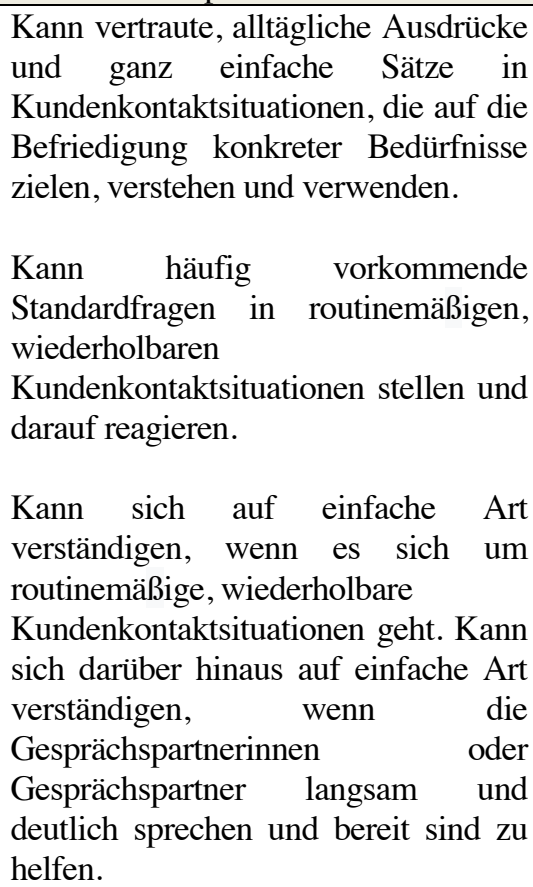 \\
\hline
\end{tabular}




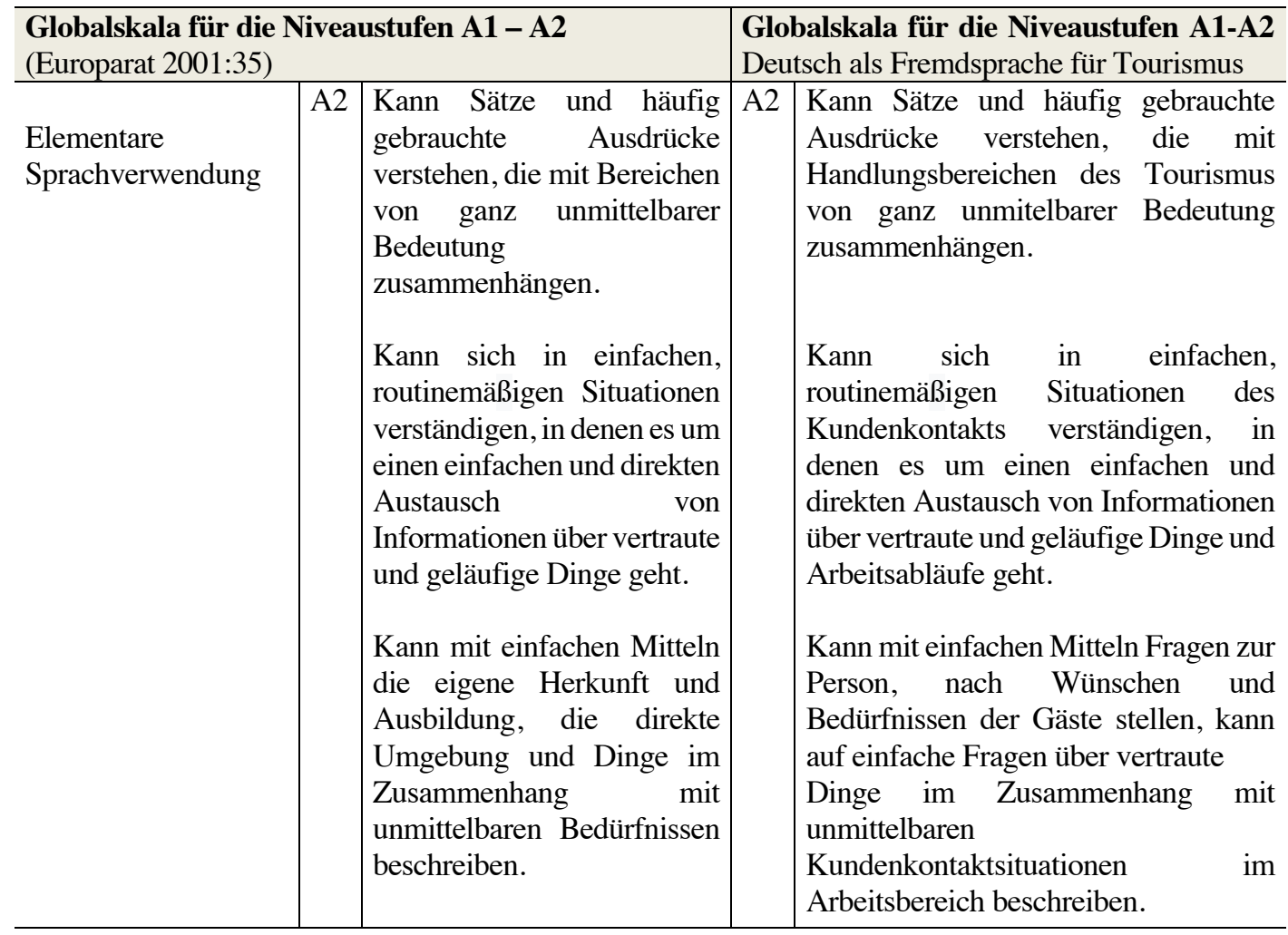

Tabelle 1. GER-Globalskala Niveaus A1-A2 und Globalskala DaFT Niveau A1-A2.

DaFT kann dementsprechend als Berufssprache angesehen werden, sofern der Bereich des Fach- bzw. Berufswortschatzes ein besonderes Teilsystem bei ihrer Vermittlung darstellt (Bosch-Roig, 2018:139). Die berufsbezogene kommunikative Kompetenz soll durch den Einsatz von Texten im weitesten Sinne und Fachund Berufswortschatz trainiert werden. Die Studierenden erweitern dabei ihre Kenntnisse der Lexik und lernen, sie im passenden beruflichen Kontext anzuwenden. Die Wortschatzvermittlung fungiert somit als ein wichtiges Mittel für die Entwicklung der berufsbezogenen kommunikativen Kompetenz im DaFT-Unterricht anhand implizit-prozeduraler sowie explizit-deklarativer Lernprozesse (Bosch-Roig, 2013a).

Aus den Überlegungen des dargestellten DaFT-Ansatzes ergeben sich für die Entwicklung einer berufsbezogenen und kommunikativen Handlungskompetenz bei der Sprachvermittlung die folgenden Teilkompetenzen (Goethe Institut, 2017: 109-130):

- Linguistische Kompetenz mit Schwerpunkt auf Fach-Berufswortschatz und Funktionsgrammatik.

- Soziolinguistische Kompetenz mit Schwerpunkt auf formellen Interaktionsformeln und Höflichkeitskonventionen.

- Pragmatische Kompetenz mit Schwerpunkt auf Diskurs- und Interaktionsstrategien.

- Funktionale Kompetenz mit Schwerpunkt auf Form- und kommunikativen Funktionszusammenhängen.

Die aufgestellten Teilkompetenzen stellen den Kern des Deutschunterrichts für Tourismus dar und werden fester Bestandteil der DaFT-Prüfungen und Tests.

\section{MOOC DaFT an der Universität der Balearen}

Die Beschäftigung in der heutigen Arbeitswelt erfordert zunehmend eine fachorientierte Berufsausbildung und Spezialisierung, weswegen Rezepte wie "Englisch für alles und jeden" nicht mehr ausreichen. Kenntnisse dieser 
Sprache sind heute eine unabdingbare Voraussetzung, obwohl es auch immer mehr Stimmen gibt, die die Beherrschung der englischen Sprache als unzureichend betrachten, um in einer wettbewerbsorientierten Wirtschaft eine qualitativ hochwertige Beschäftigung zu erreichen.

Die Ressourcen sind nicht unbegrenzt und die Lernzeit ist ebenfalls begrenzt, was die Notwendigkeit einer Optimierung dieser Komponenten erhöht. Das Angebot von Sprachen innerhalb aller akademischen Studien, auch außerhalb der akademischen Welt, sollte gewährleistet sein und auch auf einen tatsächlichen Bedarf, d.h. auf eine vorherige Analyse der unmittelbaren Umgebung, reagieren. Dieses "unmittelbare Umfeld" bezieht sich auf den engsten und direkten Kontext der Studierenden, in erster Linie auf die lokale und regionale Nachfrage, auf Unternehmen im direkten universitären Umfeld sowie auf öffentliche und private Institutionen, die an wirtschaftlichen Prozessen beteiligt und prägend sind. Wir verweisen auf eine Analyse des Sprachbedarfs, die die Nachfrage nach Sprachen in den umliegenden Wirtschaftssektoren transparenter macht.

Auf der Insel Mallorca gibt es immer mehr deutsche Unternehmen, nicht nur des Tourismus. Die deutsche Sprache ist derzeit aus diesem Grund eine sehr wichtige Arbeitssprache in diesem geografischen Gebiet. Wir müssen jedoch das knappe Ausbildungsangebot dieser Sprache im akademischen Umfeld der Universität noch einmal betonen, und dies führt zu einer paradoxen Situation: akademische Überqualifizierung zusammen mit geringer Sprachausbildung. Das derzeitige Sprachtraining der Studenten des Abschlusses in Tourismus garantiert nicht ihre Wettbewerbsfähigkeit auf dem Arbeitsmarkt der Insel. Dieser Umstand ist nicht nur auf den Balearen anzutreffen, es ist offensichtlich, dass die Ausbildung in Fremdsprachen in Spanien immer problematisch war und bleibt (Eurostat, 2019).

Der Abschluss in Tourismus und die Doppelabschlüsse in Tourismus-BWL und Tourismus-VWL, die von der Fakultät für Tourismus der Universität der Balearen angeboten werden, sehen lediglich drei obligatorische Deutschkurse für drei Semester vor, was es unmöglich macht, Studenten ausreichend für ihr berufliches Umfeld auszubilden, da es schwierig ist, ein anfängliches B1-Niveau ab einem A2-Niveau zu erreichen. Den Tourismusstudenten wird daher empfohlen, vor der Einschreibung in den ersten Deutschkurs selbst ein vollständiges A1-Niveau zu absolvieren. Diese wichtige Voraussetzung ist jedoch nicht immer erfüllt, so dass wir in den ersten Kursen Studenten ohne Vorkenntnisse in Deutsch finden können. Um dieses Problem zu lösen, haben wir einen Online-Kurs (MOOC DaFT) für Anfänger entwickelt, d.h. einen Kurs für die Stufen A1-A2, um auf diese Weise unseren Studenten den Zugang zu den Vorkenntnissen zu erleichtern, die erforderlich sind, um die späteren Pflichtfächer absolvieren zu können. Natürlich ermöglicht das Kursformat auch den Lerneinstieg jener, die bereits im Berufsleben stehen oder ihre Deutschkenntnisse auffrischen oder erweitern möchten.

\subsection{Szenarien als mikro-didaktische Lerneinheiten}

Das vorgeschlagene MOOC DaFT ist als ein Basiskurs außerhalb des Lehrplans konzipiert worden, d.h. als einführender Lernblock für Universitätsfächer, der nicht auf unbestimmte Zeit verlängert werden sollte. Er dauert zwischen 7 und 10 Wochen, damit sich unsere Studenten richtig vorbereiten können, bevor sie reguläre Kurse an der Universität beginnen.

Der DaFT-Einstiegskurs setzt sich aus folgenden Szenarien bzw. Lerneinheiten zusammen, wobei der Kontext der Sprachverwendung als beruflicher Bereich, die Situationen (Orte, soziale Rollen, Texte und Handlungen) und die Themen (Strukturen und Wortschatz) vorgegeben werden: 
Szenario 1 - Willkommen an der Fakultät für Tourismus

Szenario 2 - Im Hotel an der Rezeption

Szenario 3 - Frühstück im Hotel - Bestellen im Restaurant

Szenario 4 - In der Küche - Dialog am Büffet

Szenario 5 - Im Informationsbüro

Szenario 6 - In der Autovermietung

Szenario 7 - Am Flughafen

Die Szenarien wurden nach Relevanzkriterien ausgewählt, d.h. man versucht insbesondere, typische Kundenkontaktsituationen des Berufslebens im Tourismusbereich anhand von Interviews und Beobachtungen vor Ort zu identifizieren, die spezifische Kommunikationsbedürfnisse darstellen und eine reale Sprachverwendung widerspiegeln. Aus diesem Grund werden im MOOC reale Aufgaben in Kundenkontaktsituationen des Tourismus anhand von Videos dargestellt. Tourismusstudenten sowohl Muttersprachler als auch nicht-Muttersprachler fungieren hier als Schauspieler, denn verschiedene phonetische Realisierungen und Akzente erleichtern den Studenten in den Anfangsphasen einen angstfreieren Einstieg in die deutsche Sprache. Dass die Deutschlehrer/innen in Spanien nicht alle Muttersprachler sind, ist eine Realität, die man auch in diesem Kurs widerzuspiegeln versuchte.

Neben den genannten Videos haben die Lerner auch Zugriff auf die Transkripte der Dialoge, auf die Vokabellisten jedes Szenarios inklusive der Podcasts. Eine kurze, visuelle Grammatikerklärung anhand von Videos und eine Reihe von kurzen Übungen oder pädagogischen Aufgaben im digitalen Klassenzimmer hilft den Lernern, auf die Form zu achten, d.h. grammatikalische Formen und Strukturen nicht isoliert, sondern als Form-Funktions-Beziehungen zu üben. Eine kommunikative Basisgrammatik für den Tourismus als Zusatz- und Nachschlagematerial steht ihnen ebenfalls auf der Plattform zur Verfügung.

Eine zweite Reihe von pädagogischen Aufgaben im digitalen Klassenzimmer zielt darauf ab, die Kommunikationsfähigkeit des Studierenden Schritt für Schritt zu entwickeln.

Am Ende jedes Szenarios ist ein multiple-choice-Test vorgesehen, damit die rezeptive Aneignung von Strukturen und Wortschatz überprüft wird. In einem weiteren und abschließenden Schritt werden die Lerner aufgefordert, eine kommunikative Aufgabe in Form eines Podcasts mit einem Klassenkameraden durchzuführen. Sinn dieser Aktivität ist die praktische Umsetzung und Anwendung des Erlernten in einer interaktiven Aufgabe.

Zusammenfassend können wir an dieser Stelle festhalten, dass die Förderung der rezeptiven Fertigkeit verstehen mündlicher Texte an erster Stelle sowie das Sprechen bzw. die Interaktion den Kern unseres MOOC DaFT ausmachen. Das Design rezeptiv- und produktiv-interaktiver Aktivitäten soll diesem Ziel dienen. Im folgenden Abschnitt befassen wir uns mit ihrer Beschreibung.

\subsection{Deskriptoren rezeptiv- und produktiv-interaktiver Aktivitäten im MOOC DaFT}

Die folgenden Deskriptoren gelten für ein A1-A2 Niveau und sind in Anlehnung an die vom GER entwickelten Skalas konzipiert worden (Goethe Institut, 2017). 


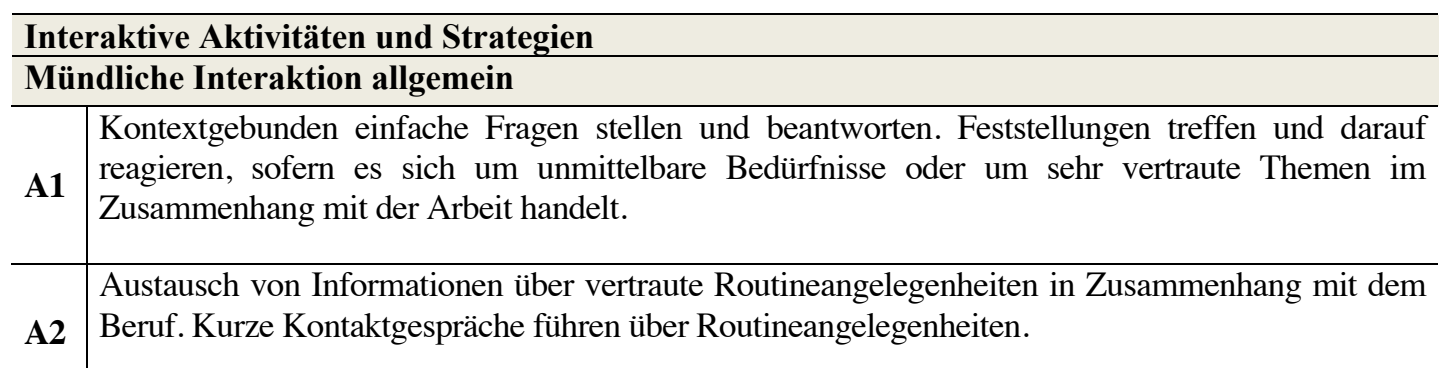

\section{Gesprächspartner verstehen}

A1 Ausdrücke verstehen, die auf die Befriedigung einfacher, konkreter Bedürfnisse der Gäste zielen. Einfache Bitten, Fragen und Anweisungen im Zusammenhang mit vertrauten Situationen verstehen.

A2 Einfache Routinegespräche kontextabhängig verstehen. Telefonate über Routineangelegenheiten annehmen, verstehen und darauf reagieren.

\section{Konversation}

A1 Gäste empfangen, sich vorstellen, einfache Gruß- und Abschiedsformeln gebrauchen. Nach dem Befinden fragen, einfache Wünsche äußern.

A2 Höflichkeitsformeln verwenden, Gäste grüßen und ansprechen, sich verabschieden, sich entschuldigen, auf Entschuldigung reagieren. Kurze Kontaktgespräche führen.

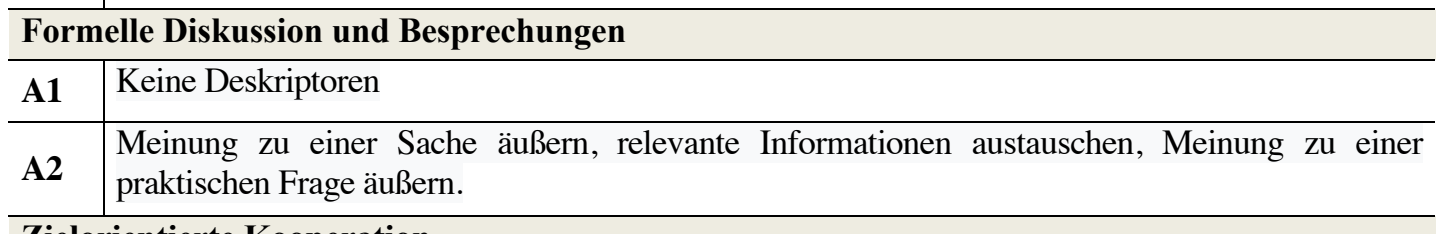

\section{Zielorientierte Kooperation}

A1 Jemanden um etwas bitten, auf einfache Bitten reagieren, jemandem etwas geben.

A2 Dinge zu erbitten oder zu geben, einfache Informationen erfragen und besprechen, was man als Nächstes tun wird.

\section{Dienstleistungsgespräche}

A1 Um etwas bitten, etwas geben, zahlen, Preise und Uhrzeiten angeben und sich darüber austauschen.

Routinemäßige Kundenkontaktsituationen des Tourismus bewältigen: Check-in u. Check-out,

A2 Gerichte erklären, Bestellungen entgegennehmen, Anweisungen geben, Mitteilungen und Empfehlungen machen.

Informationsaustausch

A1 Zeitangaben mit Hilfe von Wendungen machen, Fragen und Anweisungen der Gäste verstehen A1 und darauf reagieren, einfache Fragen stellen und beantworten, Fragen zur Person stellen.

Routinegespräche führen, unkomplizierte Sachinformationen herausfinden und weitergeben,

A2 Informationen geben und erfragen, den Weg erklären, begrenzte Informationen über routinemäßige Arbeitshandlungen austauschen.

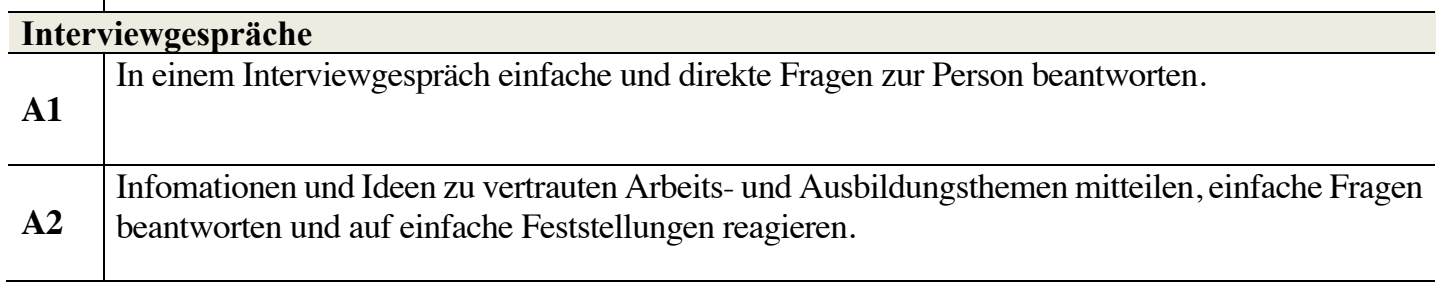

Tabelle 2. GER-Deskriptoren A1-A2 für DAFT (Goethe-Institut, 2017: 78-85). 
Für die schriftliche Interaktion gelten anhand des GER die folgenden Skalas:

\begin{tabular}{|c|c|}
\hline \multicolumn{2}{|c|}{ Schriftliche Interaktion } \\
\hline \multicolumn{2}{|c|}{ Schriftliche Interaktion allgemein } \\
\hline A1 & Schriftlich Informationen zur Person erfragen oder weitergeben. \\
\hline A2 & Kurze, einfache, formelhafte Notizen machen. \\
\hline \multicolumn{2}{|r|}{ Korrespondenz } \\
\hline A1 & Kurze E-Mails verstehen und schreiben. \\
\hline A2 & Einfache E-Mails verstehen und schreiben, sich bedanken und entschuldigen. \\
\hline \multicolumn{2}{|r|}{ Notizen, Mitteilungen und Formulare } \\
\hline A1 & $\begin{array}{l}\text { Zahlen, Daten, Namen, Nationalität Alter, Geburtsdatum, Ankunftsdatum usw. in einen } \\
\text { Anmeldezettel im Hotel oder bei der Einreise eintragen. }\end{array}$ \\
\hline A2 & $\begin{array}{l}\text { Kurze, einfache Notizen und Mitteilungen schreiben, sich auf unmittelbare Bedürfnisse beziehen, } \\
\text { kurze einfache Mitteilungen von Gästen entgegennehmen. }\end{array}$ \\
\hline
\end{tabular}

Tabelle 3. GER-Deskriptoren A1-A2 für DAFT. (Goethe-Institut, 2017:86-87).

Die oben dargestellten Deskriptoren sollen als Grundbasis für die Entwicklung eines DaFT-Tests dienen. Damit werden wir uns im nächsten Abschnitt beschäftigen.

\subsection{Sinn einer DaFT-Test-Entwicklung im Rahmen des MOOC}

An diesem Punkt angekommen, wollen wir uns die Frage nach dem Sinn eines DaFT-Tests stellen. Man könnte davon ausgehen, dass die vom GER entwickelten Deskriptoren, denen die bereits bestehenden und international anerkannten Prüfungen Start Deutsch 1 und Start Deutsch 2 (telc Deutsch A1/A2) des Goethe Instituts und der telc $\mathrm{GmbH}$ entsprechen, geeignet sind für eine Zielgruppe von Lernern, die bereits im Tourismusbereich tätig, sich weiter qualifizieren und ihre Sprachkompetenz zertifizieren lassen oder aber ihre Berufschancen auf dem Markt erhöhen, eine Umschulung machen möchte, usw. Dennoch muss man festellen, dass im Fall des telc Tests Deutsch A1/A2 (Hantschel et. al., 2010) nur sehr allgemeine Aspekte des Berufslebens berührt werden, die sich insbesondere an Migranten richten, die in Deutschland auf Arbeitssuche sind. Der Test telc Deutsch A2+ Beruf ist spezifischer, aber strebt ebenfalls das Ziel der Integration von Lernern mit Migrationshintergründen in deutschsprachigen Ländern an. Der Goethe Test Pro Deutsch für den Beruf ist ein intelligenter onlineSchnelltest, der während der Durchführung und anhand ihrer Antworten sich an das Niveau der Testler anpasst. Es handelt sich um allgemeine Inhalte des Berufslebens, insbesondere des Wirtschaftslebens und testet die Fertigkeiten Lesen und mündliches Verstehen.

Wir stellen fest, dass es keine spezifischen Prüfungen für Deutsch für den Tourismus auf dem Markt gibt, obwohl Deutsch eine der wichtigsten Tourismussprachen der Welt ist. Als Berufssprache ist Deutsch auf den Balearen insbesondere auf Mallorca zwingend erforderlich (Bosch-Roig, 2016, 2018); aus diesem Grund finden wir es besonders dringend, einen DaFT-Test zu entwickeln, der die kommunikativ- interaktiven Sprachkompetenzen der Lerner auf einem Anfängerniveau (A1/A2) online prüfen kann. Somit könnten potentielle Arbeitnehmer ein Basisniveau zertifizieren, das ihre kommunikative Handlungsfähigkeit in ganz konkreten Situationen auf einem minimalen Niveau garantiert. Für die Arbeitgeber wäre der DaFT-Test ein nützliches Instrument, um die Kenntnisse und Kompetenzen ihrer Arbeitskandidaten zielgerichtet, sachlich und autonom zu prüfen. 


\section{Schluss}

Angesichts der Nachfrage nach Deutsch für den Tourismus auf den Balearen und des geringen Ausbildungsangebotes im akademischen Bereich, gilt es Kurse zu entwerfen, die es den Studenten ermöglichen, sich autonom und flexibel aus- und weiterzubilden.

Damit sie sich Basiskenntnisse und Kompetenzen aneignen können, haben wir einen kommunikativen, aufgabenorientierten Online-Kurs an der Universität der Balearen entwickelt, in dem reale Situationen des Berufslebens auf einem Anfängerniveau geübt und bewältigt werden können.

Neben dem Leistungsnachweis über diesen Kurs ist ein DaFT-Zertifikat in Anlehnung an den GER und anhand des entwickelten Inhalts und Materials zu entwerfen, das insbesondere für die Arbeitnehmer von Nutzen sein kann. Damit wäre es möglich, von einem Basisniveau bei potentiellen Arbeitnehmern auszugehen, das ein Minimum an Kenntnissen und Kompetenzen garantieren kann.

Auf dem Markt gibt es noch keinen spezifischen DaFT-Test, der rezeptiv- und produktiv-interaktive Aktivitäten mit besonderem Nachdruck auf den Berufs- und Fachwortschatz prüft. Damit würden ganz konkrete Bedürfnisse des Berufsbildes berücksichtigt und erfüllt werden.

Unser nächste Schritt ist die Entwicklung und Validierung dieses DaFT-Tests.

\section{Declaration of conflicting interests}

The author(s) declared no potential conflicts of interest with respect to the research, authorship, and/or publication of this article.

\section{Funding}

The author(s) received no financial support for the research, authorship, and/or publication of this article.

\section{About the author}

Gloria Bosch es Profesora Titular en el área de alemán de la Universidad de las Islas Baleares. Imparte docencia en el Grado de Estudios Ingleses, en los Dobles Grados ADE- Economía y Turismo, en el Máster de Lenguas y Literaturas Modernas y en el Máster de Profesorado. Entre sus líneas de investigación destaca la didáctica y adquisición del alemán para fines específicos. Cuenta con numerosas publicaciones nacionales e internacionales en revistas y editoriales de prestigio como Walter de Gruyter, Peter Lang, Cambrigde Scholars, entre otras. También ha publicado material didáctico para Turismo en la editorial alemana Hueber, además de varios manuales de aprendizaje en la editorial de la Universidad de las Islas Baleares. Ha llevado a cabo diversos proyectos de innovación docente. 


\section{Literaturverzeichnis}

Bosch-Roig, G. (2013a). Wortschatzvermittlung durch concept mappings zur Förderung kommunikativer (Fach)Kompetenz im DaftUnterricht. Zielsprache deutsch.Eine internationale Zeitschrift für Deutsch als Fremdsprache/Deutsch als Zweitsprache, 40(3), 318.

Bosch-Roig, G. (2013b). "Aufgaben- und Inhaltsorientierung als methodologisches Konzept für ein integriertes Lernen im DaFTUnterricht auf Mallorca". In Kiefer, K. H. \& Efing, Ch., et al.(Hrsg.), Berufsfeld-Kommunikation: Deutsch, Band 7, (pp.137-148). Bern: Peter Lang.

Bosch-Roig, G. (2016). Problem Fremdsprachen: Deutsch auf Mallorca aus der Markt- und Wirtschaftsperspektive. Synergy, 12(1), 72-83.

Bosch-Roig, G. (2018). "Fremdsprachen im Tourismus: Situation und Perspektiven in Spanien”. In Ulrich A. \& Costa, M. (Hrsg.), Sociolinguistica. Ein internationales Jahrbuch für europäische Soziolinguistik 32(1), (pp.117-129). München: De Gruyter.

Curso Básico de alemán para Turismo (2020). <https.www://miriadax.net/web/curso-basico-de-aleman-para-turismo/inicio>. [30/04/2020].

Efing (2014). Berufssprache \& Co.: Berufsrelevante Register in der Fremdsprache. Ein Varietätenlinguistischer Zugang zum berufsbezogenen DaF-Unterricht. Info DaF 4, 415-441.

EUROPARAT (2001). Gemeinsamer europäischer Referenzrahmen für Sprachen: Lernen, lehren, beurteilen. Berlin: Langenscheidt.

EUROSTAT (2019). Foreign language skills statistics. <44913.pdf (europa.eu) >. [20/04/2020].

Goethe Institut (Hrsg.) (2017). Gemeinsamer europäischer Referenzrahmen für Sprachen: lernen, lehren, beurteilen. Stuttgart: Klett.

Grotjahn, R. \& Kleppin, K. (2015). Prüfen, Testen, Evaluieren. München: Klett-Langenscheidt.

Hantschel, H-J. et al. (2010). Start Deutsch 1/2 A1/A2 (telc Deutsch A1/A2). Stuttgart: Klett.

IBESTAT (2019). Flujo de Turistas (FRONTUR).

<https.www://ibestat.caib.es/ibestat/estadistiques/economia/turisme/fluxe-turistes-frontur/043d7774-cd6c-4363-929a703aaa0cb9e0>. [30/04/2021].

Klein, K. \& Hohmann, S. (2018). Mit Erfolg zu telc Deutsch B1-B2 Beruf. Stuttgart: Klett.

Long, M. (2014). Second Language Acquisition and Tasked-Based Language Teaching. New York: Wiley-Blackwell.

Loumiotis, U.\& Näfken, A. (2016). Mit Erfolg zum Goethe-Zertifikat A2. Stuttgart: Klett. 